\title{
Chirality and Wine
}

\author{
Shaun McCann ${ }^{1}$
}

Received: 18 October 2018 / Accepted: 18 October 2018 / Published online: 6 November 2018

(c) Springer Nature Limited 2018

\section{A bottle of wine contains more philosophy than all the books in the world.}

Louis Pasteur, French biologist, microbiologist and artist (1822-1895).

Penicillin cures, but wine makes you happy.

Alexander Fleming (1881-1955).

All you clever people who read this journal have undoubtedly heard of Louis Pasteur. No doubt you all know about the technique of 'pasteurization' which bears his name. This technique is predicated on Pasteur's theory that tiny organisms (germs) could cause disease and could be destroyed by heating. The technique is commonly used to kill bacteria in milk and was used (Figs. 1 and 2), but no longer, to do the same in wine making.

However, I bet you did not know that Louis Pasteur started life as an artist. Although he described 'chirality' in organic chemistry, an article by Joseph Gal in Nature [1] suggests that this observation was influenced by Pasteur's previous experience as an artist and lithographer. In lithography a drawing is etched onto limestone with wax or oil and acid and then white paper pressed on top of the etching produces the print, a mirror image of the original drawing. You are probably all familiar with stereoisomers but 'chirality' means that some molecules in mirror image are capable of producing different effects. Thus Pasteur identified the two enantiomers of a chiral substance. He presented his findings to the Académie des Sciences in 1848 and suggested that chirality or molecular dissymmetry arises from a

Shaun McCann

shaunrmccann@gmail.com

1 Department of Haematology and Academic Medicine, St James' Hospital and Trinity College, Dublin, Ireland tetrahedral or helical arrangement of atoms. I must confess that we rarely discussed chirality on 'ward rounds'; in fact, if the truth was known, we never did!

The most infamous example of how a chiral drug can cause teratogenicity is the drug Thalidomide [2]. This drug which was one of the largest selling drugs in the late 1950s, was very effective in treating 'morning sickness' of pregnancy. As well as causing a peripheral neuropathy the drug produced teratogenic effects notably phocomelia. The precise mechanism is unclear but may be related to its antiangiogenic effect. It seems that thalidomide is chiral and the teratogenic effect, which is species specific, is caused by the $\mathrm{S}$ enantiomer. The $\mathrm{R}$ enantiomer inhibits cereblon which is part of the e3 ubiquitin ligase complex. The drug oscillates between enantiomeric states and it is very difficult to make a stable form that is non-teratogenic [3]. The drug never received FDA approval; however, analogues of thalidomide are now widely used to treat multiple myeloma and plasma cell myeloma [4]. Other chiral drugs familiar to haematologists are cyclosporine and MMF (mycophenolic acid). The relationship between the chirality of the anti-malarial drug mefloquine and the intracellular concentrations of cyclosporine is illustrated by examining the effects of enantiomers of mefloquine on the transport activity of $\mathrm{P}$ glycoprotein (Pgp) $(+)$-mefloquine competitively displaces the Pgp substrate cyclosporine whereas the (-) mefloquine has no effect on cyclosporine-Pgp binding. William SA Knowles, Bary Sharpless and Ryori Nyori were awarded the Nobel Prize in Chemistry in 2001 for their development of asymmetric synthesis using chiral catalysts in the production of single enantiomer drugs or chemicals.

Two acids that are found in wine are tartaric and malic acid. Malic acid is converted into lactic acid during malolactic fermentation by lactobacillus O.Oeni. The more correct term is malolactic conversion. Malic acid is converted into the less acidic lactic acid and carbon dioxide. Although this phenomenon occurs naturally in most vineyards some oenologists may add cultured lactobacilli to achieve this conversion. The chardonnay grape is particularly prone to malolactic conversion and this phenomenon is 


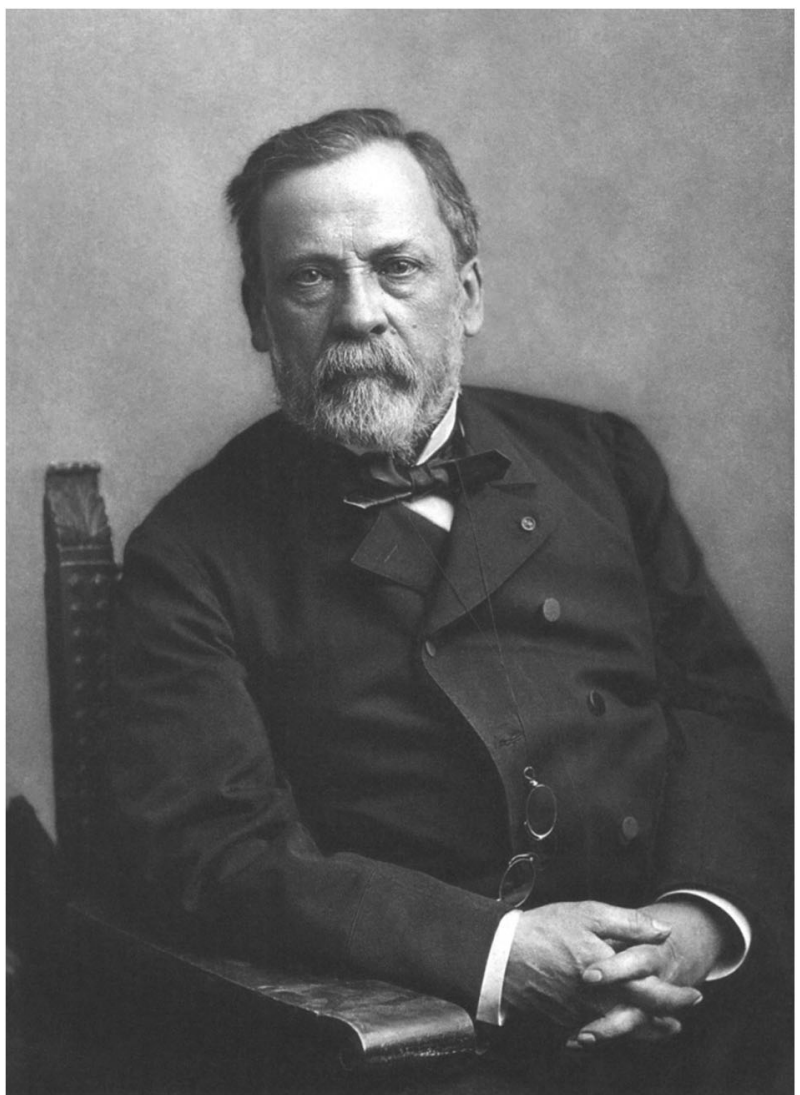

Fig. 1 Louis Pasteur

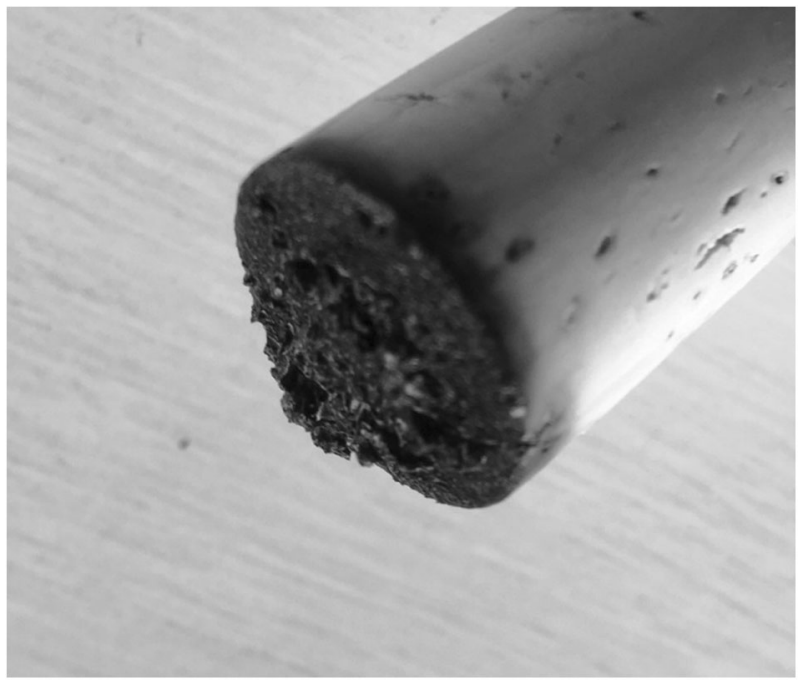

Fig. 2 Tartrate crystals on a cork from a bottle of Saint Julien 2010 reputed to give white wines from Burgundy that beautiful 'buttery' taste. Tartaric acid helps to give wine its 'tartiness' although the concentration may vary in different grape varieties. The grapes used to make sherry (palomino) have high concentrations of tartaric acid whereas pinot noir grapes contain little tartrate. Tartaric acid binds with potassium to form crystals that are tasteless and totally harmless. These crystals can be removed prior to bottling by 'cold stabilization' because some wine makers are afraid that they may be mistaken for glass shards. If not removed tartrate crystals are seen on the cork and can be removed by decanting.

Louis Pasteur found that there were two forms of tartrate crystals, mirror images of each other and said that the molecular structure of these crystals was chiral. Gal suggests that this observation was influenced by his prior artistic endeavours. So things that don't seem to be connected, art, biochemistry and wine making may, in fact, be closely related.

Acknowledgements I thank Professor Robert Peter Gale for the information on the drug mefloquine.

\section{Compliance with ethical standards}

Conflict of interest The author declares that he has no conflict of interest.

\section{References}

1. Gal G. Pasteur and the art of Chirality. Nat Chem. 2017;9:604-5.

2. Vargesson N. Thalidomide-induced teratogenesis: history and mechanisms. Birth Defects Res C Embryo Today. 2015;105:140 56.

3. Donovan KA, An J, Nowak RP, Yuan JC, Fink EC, Berry BC et al. Thalidomide promotes degradation of SALL4, a transcription factor implicated in Duane Radial Ray syndrome. eLife. 2018;7:e38430 https://doi.org/10.7554/eLife.38430.

4. Rehman W, Arfons LM, Lazarus HM. The rise, fall and subsequent triumph of thalidomide: lessons learned in drug development. Ther Adv Hematol. 2011;2:291-308. 